\title{
Efficacy and safety of concurrent anti- Cancer and anti-tuberculosis chemotherapy in Cancer patients with active Mycobacterium tuberculosis: a retrospective study
}

Tomonori Hirashima1*, Yoshitaka Tamura², Yuki Han, Shoji Hashimoto³, Ayako Tanaka', Takayuki Shiroyama', Naoko Morishita', Hidekazu Suzuki ${ }^{1}$, Norio Okamoto ${ }^{1}$, Shinobu Akada ${ }^{4}$, Makoto Fujishima ${ }^{5}$, Yoshihisa Kadota ${ }^{6}$, Kazuya Sakata ${ }^{7}$, Akiko Nishitani ${ }^{7}$, Satoru Miyazaki ${ }^{7}$ and Takayuki Nagai ${ }^{3}$

\begin{abstract}
Background: In our previous study, colorectal cancer (CRC) patients with active Mycobacterium tuberculosis (MTB) tolerated concurrent anti-cancer chemotherapy (anti-CCT) and anti-MTB chemotherapy. In this study, we retrospectively confirmed the efficacy and safety of concurrent chemotherapy in a greater number of patients with different types of malignancies.
\end{abstract}

Methods: We enrolled 30 patients who were treated concurrently with anti-CCT and anti-MTB regimens between January 2006 and February 2016. Cancer and MTB treatments were administered according to the approved guidelines.

Results: Patient demographics included: men/woman: 24/6; median age: 66.5 years; Eastern Cooperative Oncology Group performance status 0-1/2/3-4: 24/4/2; Stage IIB-IIIC/IV/recurrence: 6/22/2; lung cancer (LC)/CRC/other: 15/10/5; and MTB diagnosis (before or during anti-CCT): 20/10 (LC: 8/7; CRC: 8/2; other: 4/1). For anti-CCT, 23 patients received two cytotoxic agents with or without targeted agents and 7 patients received a single cytotoxic or targeted agent. The overall response rate was $36.7 \%$. Regarding anti-MTB chemotherapy, 22 patients received a daily drug combination containing isoniazid, rifampicin, and ethambutol, plus pyrazinamide in 15 of the 22 patients, followed by daily isoniazid and rifampicin; the remaining 8 patients received other combinations. Hematological adverse events of Grade $\geq 3$ were observed in 19 (67.9\%) of 28 patients; laboratory data were lost for the remaining 2. Grade 3 lymphopenia and higher were significantly more frequent in LC compared to other malignancies $(P<0.005)$. Non-hematological adverse events of Grade $\geq 3$ were observed in 5 (16.7\%) of 30 patients. One CRC patient experienced Grade 3 hemoptysis and another 2 experienced Grade 3 anaphylaxis. One patient with cholangiocellular carcinoma and gastric cancer experienced Grade 3 pseudomembranous colitis as a result of a Clostridium difficile infection. One patient (3.3\%) died of pemetrexedinduced pneumonitis. The success of the anti-MTB chemotherapy was 70.0\%. There were no MTB-related treatment failures. The median overall survival (months, 95.0\% confidence interval) was 10.5 (8.7-36.7), 8.7 (4.7-10.0), 36.7 (minimum 2.2), and 14.4 (minimum 9.6) for all patients combined, LC, CRC, and Other malignancies, respectively. LC patients experienced delayed MTB diagnosis and shorter overall survival.

(Continued on next page)

\footnotetext{
* Correspondence: hirashimat@ra.opho.jp

1Department of Thoracic Oncology, Osaka Habikino Medical Center, 3-7-1

Habikino, Habikino City, Osaka 583-8588, Japan

Full list of author information is available at the end of the article
}

(c) The Author(s). 2018 Open Access This article is distributed under the terms of the Creative Commons Attribution 4.0 International License (http://creativecommons.org/licenses/by/4.0/), which permits unrestricted use, distribution, and reproduction in any medium, provided you give appropriate credit to the original author(s) and the source, provide a link to the Creative Commons license, and indicate if changes were made. The Creative Commons Public Domain Dedication waiver (http://creativecommons.org/publicdomain/zero/1.0/) applies to the data made available in this article, unless otherwise stated. 
(Continued from previous page)

Conclusions: Concurrent chemotherapy is effective and safe for treating cancer patients with active MTB.

Keywords: Concurrent chemotherapy, Tuberculosis, Breast cancer, Colorectal cancer, Efficacy, Gastric cancer, Lung cancer, Safety

\section{Background}

Mycobacterium tuberculosis (MTB) represents the leading cause of death from an infectious disease worldwide [1], with the majority of cases occurring in Asia (61.0\%) and Africa (26.0\%). Incidence and mortality rates are noticeably higher in Japan than other developed countries [1].

Although active MTB infections may be present in cancer patients, our previous preliminarily report [2] is the only study to discuss the clinical course and chemotherapy outcomes of concurrent anti-cancer chemotherapy (anti-CCT) and anti-MTB chemotherapy, revealing that patients with metastatic colorectal cancer (CRC) and active MTB could safely and effectively continue anti-CCT, and achieve comparable survival to those without the infection, upon receiving appropriate MTB treatment [2].

In this study, we retrospectively examined the clinical course and chemotherapy outcomes of a larger number of patients treated concurrently with anti-CCT and anti-MTB chemotherapy.

\section{Methods}

\section{Study approval}

The present retrospective study was approved by the Institutional Review Board (IRB) of the Osaka Habikino Medical center on 30 January 2017 (approval number: 808-1). The board waived the requirement for informed written consent due to the anonymous nature of the data, and no risk of exposure to subjects.

\section{Patient selection}

We enrolled 30 cancer patients with active $M T B$ who were treated concurrently with anti-CCT and anti-MTB chemotherapy at our institution between January 1, 2006 and February 29, 2016. The 6 metastatic CRC patients with active $M T B$ from our previous study [2] were also included.

\section{Clinical review}

As described previously, [2] the clinical history of eligible patients was retrospectively reviewed. We collected baseline demographic data and anti-CCT data, which were also collected from clinical records or pharmacy database. Complete history and physical examinations; surgical reports; findings of flexible bronchoscopy, colonoscopy, and esophagogastroduodenoscopy; imaging investigations; pathological reports, and blood test results were available for all patients at the time of anti-MTB chemotherapy.

\section{MTB diagnosis}

As described previously [2], MTB diagnosis was performed by smears and cultures of various patients' specimens or chest computed tomography (CT) image in patients without microbiological evaluation. The method of choice for confirming MTB infection was Ziehl-Neelsen staining of sputum smear samples [3]. Polymerase chain reaction (PCR) or loop-mediated isothermal amplification (LAMP) [4] was performed for patients with positive sputum smears to distinguish $M T B$ from other mycobacteria. If the sputum smears were negative or specimens other than sputum were obtained, the diagnosis of $M T B$ was confirmed by culturing mycobacterial organisms. Drug sensitivity was determined for all cases with positive culture. Liquid media with the Mycobacteria Growth Indicator Tube (MGIT) [5] and solid media with the Ogawa-Kudoh method [6] were both used for culturing mycobacteria. Drug sensitivity was determined for all cases. Quantitative drug susceptibility testing for $M T B$ was performed using the MTB- $\mathrm{I}^{\oplus}$ (Kyokuto Pharmaceutical Industrial Co., Ltd., Tokyo, Japan) modified Minimum Inhibitory Concentration method [7].

\section{Treatment of MTB infection}

As described previously [2], in cancer patients with active $M T B$, appropriate anti-MTB agents were administered for approximately 1.5 months prior to anti-CCT, according to the American Thoracic Society and Infectious Diseases Society of America guidelines [8] A multi-drug resistant $M T B$, based on the sensitivity test, negated the initiation of anti-CCT. Since MTB patients with severe complications often require longer courses of treatment than those without such complications, patients treated with anti-CCT also received long-term treatment for MTB. Thus, the patients received 2 months of isoniazid $(\mathrm{H})$, rifampicin (R), ethambutol (E), and pyrazinamide (Z), followed by $H R$ for 7 months, or 6 months of HRE, followed by HR for 6 months as standard anti-MTB chemotherapy. The majority of patients who could not be treated with standard anti-MTB chemotherapy, due to side effects or drug resistance, were treated with a levofloxacin $(\mathrm{X})$-based regimen.

\section{Follow-up MTB culture}

After commencing anti-MTB chemotherapy, sputum specimens were cultured every other week for the first 
3 months. Once two consecutive sputum cultures were negative, cultures were done monthly until the course of $M T B$ treatment was completed.

\section{Definition of MTB treatment outcomes}

MTB treatment outcomes were based on the World Health Organization's definitions [9]. Treatment success included "cure" and "treatment completed". A "cured" patient was defined as one who had completed the planned treatment and had two consecutive negative cultures. Patients with treatment failure had positive cultures $\geq 5$ months into $M T B$ treatment.

\section{Cancer staging and treatment}

Cancer staging and treatment were performed according to the guidelines of the respective lung cancer (LC) [10], CRC [11], gastric cancer (GC) [12], breast cancer, [13], and cholangiocellular carcinoma (CCA) [14] societies.

The policy of our institution was to commence anti-CCT immediately if patients were sensitive to anti-MTB agents. However, if patients were resistant or had experienced intolerable side effects while awaiting sensitivity test, anti-CCT was suspended until a decision could be reached concerning the appropriate drug combination and duration of anti-MTB chemotherapy. Additionally, when MTB cultures could not be obtained, due to a number of reasons, or the physician could not await the results of the sensitivity test due to rapid tumor progression, anti-CCT was commenced, following informed patient consent.

\section{Assessment of anti-cancer chemotherapy outcomes}

Patients' best response to chemotherapy was collected from the records of weekly meetings at our institution and clinical summaries. Based on the Response Evaluation Criteria in Solid Tumors [15], the responses of tumors to cytotoxic agents were categorized as complete or partial responses and stable or progressive disease. Tumor responses that could not be assessed were recorded as "not evaluable". Adverse events were graded according to the National Cancer Institute Common Toxicity Criteria, version 3.0 [16].

\section{Statistical analyses}

Overall survival (OS) was measured from the date of commencing concurrent chemotherapy (for both cancer and MTB) to the date of death or last follow-up (February 28, 2017). OS rates were estimated using the Kaplan-Meier method [17]. The duration of concurrent chemotherapy was defined as the time from commencing concurrent chemotherapy to the end date. All statistical analyses were conducted using $R$ statistical software (version 3.2.0). Patient background data were analyzed using chi-square and Fisher's exact test for categorical variables. A $P<0.05$ was considered statistically significant.

\section{Results \\ Patient demographics}

In total, 30 cancer patients with active $M T B$ who were treated concurrently with anti-CCT and anti-MTB chemotherapy between January 1, 2006 and February 29, 2016 at our institution, were enrolled in this study. Fifteen patients were diagnosed with LC (non-small cell LC [NSCLC, $n=$ 10] and small cell LC [SCLC, $n=5]$ ), 10 patients were diagnosed with CRC (rectal cancer [ $n=7]$, sigmoid cancer $[n=2]$, and transverse colon cancer $[n=1]$ ), and 5 patients were diagnosed with other malignancies (GC $[n=1]$, Breast Cancer $[n=1]$, LC with GC $[n=1]$, CRC with GC $[n=1]$, and CCA with GC $[n=1])$. The patient demographics are summarized in Table 1. No significant differences in patient demographics were observed among LC, CRC, and other malignancy groups. However, LC patients tended to be more frequently diagnosed with active $M T B$ during anti-CCT in comparison to those with CRC or other malignancy.

\section{Chest CT imaging}

Thoracic CT findings at the time of $M T B$ diagnosis is presented in Fig. 1 (a-d). These CT images present a combination of thick and thin walled lung cavities, infiltration shadows, and multiple nodules. There were no consistent findings on CT imaging based on the type of malignancy.

\section{Bacteriological examinations}

The findings of bacteriological examinations are summarized in Table 2 . Twenty (66.7\%) patients had MTB-positive sputum smears. In $25(83.3 \%)$ cultures were positive for $M T B$ and negative cultures were reported in 5 (16.7\%). Cultures were negative for MTB in $3 \mathrm{LC}$ and 2 CRC patients. In the former, active $M T B$ was diagnosed by PCR of sputum in 2 cases and LAMP of sputum in 1, alongside CT results, while in the latter, the diagnosis was confirmed by PCR of sputum in one, and the results of the CT scan and clinical course in the other.

In the 25 cases positive for $M T B, 22$ (88.0\%) were sensitive, 2 (8.0\%) were resistant (one MTB strain was resistant to $\mathrm{H}$ and streptomycin, another to $\mathrm{H}$ and $\mathrm{Z}$ ), and $1(4.0 \%)$ was not tested for sensitivity because of concomitant Nontuberculous Mycobacteriosis. In 2 of 25 patients with $M T B$-positive cultures, the time to $M T B$-positive culture could not be confirmed because the culture specimens were obtained in other institutes. Therefore, the time to MTB-positive culture in 23 patients was demonstrated by MGIT $(n=18)$ and Ogawa-Kudoh method $(n=5)$. The time (days: mean \pm $\mathrm{SD)}$ to $M T B$-positive cultures was significantly shorter 
Table 1 Patient Characteristics

\begin{tabular}{|c|c|c|c|c|}
\hline \multirow[t]{3}{*}{ Characteristic } & \multicolumn{4}{|l|}{ Patients } \\
\hline & All & LC & CRC & Other \\
\hline & $(n=30)$ & $(n=15)$ & $(n=10)$ & $(n=5)$ \\
\hline \multicolumn{5}{|l|}{ Sex, n (\%) } \\
\hline M & $24(80.0)$ & $12(80.0)$ & $8(80.0)$ & $4(80.0)$ \\
\hline $\mathrm{F}$ & $6(20.0)$ & $3(20.0)$ & $2(20.0)$ & $1(20.0)$ \\
\hline Age (years), median (range) & $66.5(39-79)$ & $68.0(43-79)$ & $62.5(43-75)$ & $56.0(39-75)$ \\
\hline \multicolumn{5}{|l|}{ ECOG PS, n (\%) } \\
\hline $0-1$ & $24(80.0)$ & $12(80.0)$ & $8(80.0)$ & $4(80.0)$ \\
\hline 2 & $4(13.3)$ & $3(20.0)$ & $0(0.0)$ & $1(20.0)$ \\
\hline 3 & $2(6.7)$ & $0(0.0)$ & $2(20.0)$ & $0(0.0)$ \\
\hline \multicolumn{5}{|l|}{ Stage, n (\%) } \\
\hline$\|B-\| A$ & $3(10.0)$ & $2(13.3)$ & $0(0.0)$ & $1(20.0)$ \\
\hline$\||| B-\| C$ & $3(10.0)$ & $2(13.3)$ & $0(0.0)$ & $1(20.0)$ \\
\hline IV & $22(73.3)$ & $10(66.7)$ & $9(90.0)$ & $3(60.0)$ \\
\hline Postoperative recurrence, n (\%) & $2(6.7)$ & $1(6.7)$ & $1(10.0)$ & $0(0.0)$ \\
\hline \multicolumn{5}{|c|}{ Line of cancer chemotherapy at the commencement of concurrent chemotherapy, $\mathrm{n}$ (\%) } \\
\hline Adjuvant & $1(3.3)$ & $0(0.0)$ & $0(0.0)$ & $1(20.0)$ \\
\hline First & $21(70.0)$ & $9(60.0)$ & $8(80.0)$ & $4(80.0)$ \\
\hline Second & $3(10.0)$ & $3(20.0)$ & $0(0.0)$ & $0(0.0)$ \\
\hline Third or higher & $5(16.7)$ & $3(20.0)$ & $2(20.0)$ & $0(0.0)$ \\
\hline \multicolumn{5}{|l|}{ Diabetes mellitus, $\mathrm{n}(\%)$} \\
\hline Y & $7(20.7)$ & $3(20.0)$ & $2(20.0)$ & $2(40.0)$ \\
\hline $\mathrm{N}$ & $23(79.3)$ & $12(80.0)$ & $8(80.0)$ & $3(60.0)$ \\
\hline \multicolumn{5}{|l|}{ MTB diagnosis, n (\%) } \\
\hline Before anti-CCT & $20(66.7)$ & $8(53.3)$ & $8(80.0)$ & $4(80.0)$ \\
\hline During anti-CCT & $10(33.3)$ & $7(46.7)$ & $2(20.0)$ & $1(20.0)$ \\
\hline
\end{tabular}

Abbreviations CCT cancer chemotherapy, CRC colorectal cancer, ECOG Eastern Cooperative Oncology Group, $F$ female, LC lung cancer, $M$ male, MTB Mycobacterium tuberculosis, $N$ no, $P S$ performance status, $Y$ yes

for MGIT than for the Ogawa-Kudoh method (16.2 \pm 8.7 vs $37.6 \pm 8.4 ; P<0.0005$ ). Furthermore, the time (days: mean $\pm \mathrm{SD}$ ) to sensitivity testing in 22 patients except one with concomitant Nontuberculous Mycobacteriosi was $12.6 \pm 3.5$.

\section{MTB treatment until concurrent chemotherapy}

Of the 30 patients enrolled in this study, 4 received $\mathrm{X}$-based regimen from the start of $M T B$ treatment due to resistant to INH $(n=2)$, past history of RFP-induced systemic eruption $(n=1)$, and preventing the drug interaction between erlotinib and RFP via CYP3A4 $(n=1)$.

In 2 of 4 patients the X-based regimen was changed to a different regimen due to renal dysfunction in one and liver dysfunction in the other.

In 4 patients the planned HREZ or HRE regimen was changed to $\mathrm{X}$-based regimen due to drug eruption $(n=1)$, drug eruption and liver dysfunction $(n=1)$, thrombocytopenia $(n=1)$, and preventing the drug interaction between Paclitaxel and RFP via CYP3A4 and CYP2C8 $(n=1)$. Subsequently, 8 patients received concurrently $\mathrm{X}$-based regimens and anti-CCT. The remaining 22 patients received concurrent HREZ or HRE and anti-CCT as planned, without many adverse events except in one patient who experienced a paradoxical response on day 23 after starting HREZ and was kept on HREZ while taking prednisolone. Thus, until concurrent chemotherapy, $6(20.0 \%)$ of the 30 patients experienced adverse events with $M T B$ treatment alone, and in 5 the planned $M T B$ treatment was changed to other regimens.

\section{Outcomes of concurrent chemotherapy}

Details of the initial concurrent anti-CCT regimens are listed in Table 3. Twenty-three patients (76.7\%) received intensive anti-CCT regimens with or without targeted agents, 5 patients (16.7\%) received a single cytotoxic agent, and 2 patients $(6.7 \%)$ received a single targeted agent. The overall response rates (ORRs) were 36.7, 33.3 


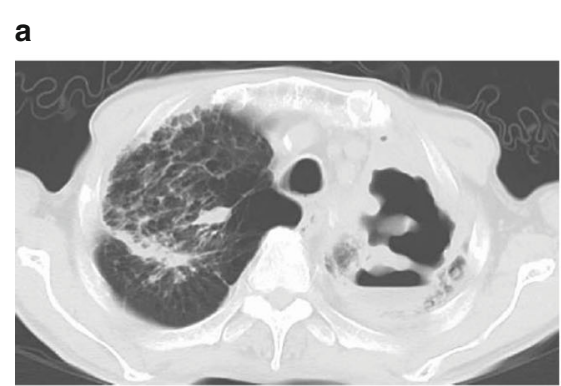

C

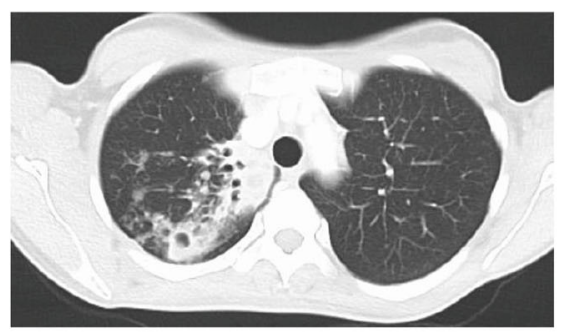

b

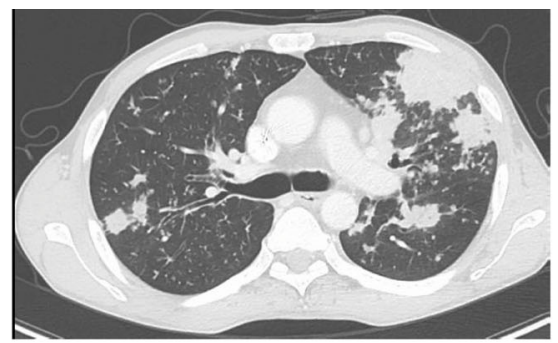

d

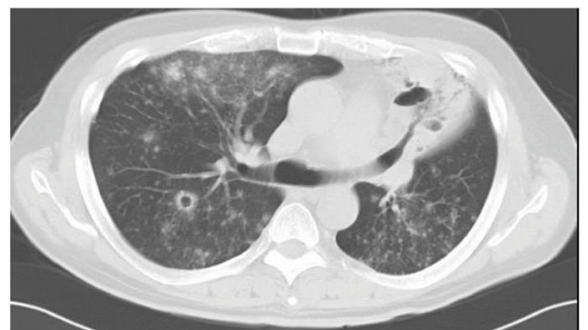

Fig. 1 Thoracic computed tomography findings in 4 cancer patients with active Mycobacterium tuberculosis. a Non-small cell lung cancer patient with a thick wall cavity in the left lung with an infiltration shadow in the right upper lobe. b Colorectal cancer patient with multiple nodules in both lungs. $\mathbf{c}$ Breast cancer patient with a small cavity with an infiltration shadow in the right upper lobe. $\mathbf{d}$ Non-small cell lung cancer patient with multiple small nodules with partial patty follicular spot in the both lungs and cavity formation in the $S 6$ segment of the right lung and lingular segment of the left lung

40.0, and 40.0\% for all patients combined, LC patients, CRC patients, and patients with other malignancies, respectively. In 15 LC patients, all 5 patients with SCLC received intensive cytotoxic treatment (carboplatin plus etoposide) as first line chemotherapy (Table 3) with a high response rate (80\%). Of 10 NSCLC patients, 4 (40\%) received platinum doublets as intensive first regimen, and one of them achieved partial response. In the remaining 6 patients $(60 \%)$, one received platinum doublets, three were administered single cytotoxic agent, and two received erlotinib as re-challenge regimen. They received those regimens as second-line or later anti-CTT and there was no responder.

The main anti-MTB regimens were as follows: HREZ/ HR ( $n=15$ patients; LC $[n=8]$, CRC $[n=4]$, other $[n=$ $3]) ; \mathrm{HRE} / \mathrm{HR}$ ( $n=7$ patients; LC [ $n=2]$, CRC [ $n=4]$, other $[n=1])$; and X-based ( $n=8$ patients; LC $[n=5]$. and CRC $[n=2]$, other $[n=1])$. The median duration (range) of MTB treatment was 275.0 (72-637), 274.0 (90-469), 259.0 (72-539), and 368.0 (273-637) days for all patients combined, those with LC, CRC, and other malignancies, respectively. The success of anti-MTB chemotherapy in each of the above groups was 70.0, 66.7, 70.0, and 80.0\%, respectively. There were no $M T B$-related treatment failures. The median duration (range) of concurrent chemotherapy was 157.5 (13-408), 117.0 (20-245), 168.5 (13408), and 155.0 (51-376) days for all patients combined, those with LC, CRC, and other malignancies, respectively.
In $20(66.7 \%)$ patients $M T B$ was cured, one $(3.3 \%)$ completed the course for MTB treatment, and 9 (30\%) died while receiving $M T B$ treatment. Among the 9 patients who died, one died of pemetrexed-induced pneumonitis and 8 died of cancer.

In 2 CRC patients with performance status of 3, anti-CCT was started because of patients' insistence and after the approval of the Cancer Board. In one of these patients, $M T B$ was resistant to $\mathrm{H}$ and $\mathrm{Z}$. Hence, $\mathrm{X}+$ Streptomycin was administered, alongside Folnic acid, fluorouracil and irinotecan for 1 cycle. The patient died 54 days after treatment withdrawal. Regular MTB cultures performed every other week were negative for six consecutive times. The other patient received modified regimen of Folinic acid, fluorouracil and oxaliplatin for 4 cycles and died 57 days after treatment withdrawal.

Epidermal growth factor receptor sensitive mutation was found in $2 \mathrm{MTB}$-positive patients with lung adenocarcinoma. They received erlotinib as re-challenge regimen alongside HRE regimen in one patient and X-based regimen without rifampicin in the other. Both died because of cancer progression, 140 and 90 days after the initiation of erlotinib, respectively. Regular MTB cultures were performed for both patients every other week. Cultures were negative in each patient two and three consecutive times, respectively. None of the 30 patients enrolled in this study experienced recurrence of $M T B$ through their clinical course. 
Table 2 Bacteriological Examinations

\begin{tabular}{|c|c|c|c|c|}
\hline \multirow[t]{3}{*}{ Characteristic } & \multicolumn{4}{|l|}{ Patients } \\
\hline & All & LC & CRC & Other \\
\hline & $(n=30)$ & $(n=15)$ & $(n=10)$ & $(n=5)$ \\
\hline \multicolumn{5}{|l|}{ Sputum smear, n (\%) } \\
\hline Negative & $10(33.3)$ & $5(33.3)$ & $3(30.0)$ & $2(40.0)$ \\
\hline Positive & $20(66.7)$ & $10(66.7)$ & $7(70.0)$ & $3(60.0)$ \\
\hline \multicolumn{5}{|l|}{ PCR/LAMP, n (\%) } \\
\hline Negative & $1(3.3)$ & $1(6.7)$ & $0(0.0)$ & $0(0.0)$ \\
\hline Positive & $15(50.0)$ & $10(66.7)$ & $3(30.0)$ & $2(40.0)$ \\
\hline Unknown & $14(46.7)$ & $4(26.7)$ & $7(70.0)$ & $3(60.0)$ \\
\hline \multicolumn{5}{|l|}{ Culture, n (\%) } \\
\hline Negative & $5(16.7)$ & $3(20.0)$ & $2(20.0)$ & $0(0.0)$ \\
\hline Positive & $25(83.3)$ & $12(80.0)$ & $8(80.0)$ & $5(100.0)$ \\
\hline \multicolumn{5}{|l|}{ Sensitivity to anti-MTB agents, n (\%) } \\
\hline Sensitive & $22(88.0)$ & $12(100.0)$ & $6(75.0)$ & $4(80.0)$ \\
\hline Resistant & $2(8.0)^{a}$ & $0(0.0)$ & $2(25.0)$ & $0(0.0)$ \\
\hline Unknown & $1(4.0)^{b}$ & $0(0.0)$ & $0(0.0)$ & $1(20.0)^{b}$ \\
\hline \multicolumn{5}{|c|}{ Time until the result of Bacteriological Examination come out } \\
\hline & Overall & \multicolumn{2}{|c|}{ Ogawa-Kudoh } & MGIT \\
\hline Time to MTB-positive culture (days), mean $\pm S D, n$ & $\begin{array}{l}20.9 \pm 12.4 \\
\left(n=23^{c}\right)\end{array}$ & \multicolumn{2}{|c|}{$\begin{array}{c}37.6 \pm 8.4 \\
(n=5)\end{array}$} & $\begin{array}{l}16.2 \pm 8.7^{*} \\
(n=18)\end{array}$ \\
\hline Time to sensitivity testing (days), mean $\pm S D, n$ & $\begin{array}{l}12.6 \pm 3.5 \\
\left(n=22^{d}\right)\end{array}$ & & & \\
\hline
\end{tabular}

Abbreviations CRC colorectal cancer, LAMP loop-mediated isothermal amplification, LC lung cancer, MGIT Mycobacteria Growth Inhibitor Tube, MTB = Mycobacterium tuberculosis, $P C R$ polymerase chain reaction, NTM Nontuberculous Mycobacteriosis

${ }^{*} P<0.0005$

${ }^{a}$ One MTB strain was resistant to isoniazid and streptomycin, another was isoniazid and pyrazinamide

${ }^{\mathrm{b}}$ One specimen with MTB-positive cultures and concomitant NTM was not used for sensitivity testing

In two specimens, measure of the time to MTB-positive cultures were not possible because the culture specimens were obtained in other institutes

${ }^{\mathrm{d}}$ Twenty-two cultures except one which was concomitant NTM and two which was obtained in other institute

\section{Adverse events of concurrent chemotherapy}

Hematological adverse events of Grade $\geq 3$ were observed in $19(67.9 \%)$ of 28 patients; the laboratory data for 2 patients were lost. Non-hematological adverse events of Grade $\geq 3$ were observed in $5(17.9 \%)$ of 28 patients.

Number of cases in concurrent chemotherapy-related adverse events are shown in Table 4. There were no significant differences in the occurrence of adverse events between different cancer types, except for lymphopenia and neutropenia. Grade 3 lymphopenia and higher were significantly more frequent in LC compared to other malignancies $(P<0.005)$. Grade 3 Neutropenia and higher tended to be more frequent in LC compared to other malignancies. In non-hematological adverse events, Grade 1-2 liver dysfunction was frequently observed in each malignancies.

One CRC patient experienced Grade 3 hemoptysis and another 2 experienced Grade 3 anaphylaxis (oxaliplatin-induced $[n=1]$ and cetuximab-induced $[n=1])$. One patient with CCA and GC experienced Grade 3 pseudomembranous colitis as a result of a Clostridium difficile infection. One LC patient died of pemetrexed-induced pneumonitis (Grade 5).

\section{Overall survival}

The median OS in all patients was 10.5 (8.7-36.7; 95.0\% confidence interval) months (Fig. 2a). The median OS according to the type of malignancy were 8.7 (4.7-10.0), 36.7 (minimum 2.2), and 14.4 (minimum 9.6) months for LC, CRC, and other malignancies, respectively (Fig. 2b).

\section{Discussion}

This study demonstrates the efficacy and safety of concurrent anti-CCT and anti-MTB chemotherapy for cancer patients with active $M T B$, confirming the findings of our previous preliminary study [2] in a bigger sample size with a variety of cancer types.

In Japan, the national rates for success, failed, died, and lost to follow-up of anti-MTB chemotherapy in 2015 were $52.8,0.4,17.0$, and $5.6 \%$, respectively [18]. In Nagoya which is almost similar to Osaka, where our institution is located, the rates of success, failed, died, and the others were 52.0, 
Table 3 Outcomes of Anti-Cancer Chemotherapy (Anti-CCT) and Anti-MTB Chemotherapy

\begin{tabular}{|c|c|c|c|c|}
\hline \multirow[t]{3}{*}{ Characteristic } & \multicolumn{4}{|l|}{ Patients } \\
\hline & $\overline{\text { All }}$ & LC (SCLC/NSCLC) & CRC & Other \\
\hline & $(n=30)$ & $(n=15,5 / 10)$ & $(n=10)$ & $(n=5)$ \\
\hline \multicolumn{5}{|l|}{ Initial concurrent anti-CCT regimen, $\mathrm{n}$} \\
\hline Intensive cytotoxic regimen and targeted agent ${ }^{\mathrm{a}}$ & 4 & 0 & 3 & 1 \\
\hline Intensive cytotoxic regimen ${ }^{\mathrm{b}}$ & 19 & $10(5 / 5)$ & 6 & 3 \\
\hline Single targeted agent ${ }^{c}$ & 2 & $2(0 / 2)$ & 0 & 0 \\
\hline Single cytotoxic agent ${ }^{d}$ & 5 & $3(0 / 3)$ & 1 & 1 \\
\hline \multicolumn{5}{|c|}{ Best response on anti-CCT after commencing concurrent chemotherapy, $\mathrm{n}$} \\
\hline$C R$ & 1 & 0 & 1 & 0 \\
\hline PR & 10 & $5(4 / 1)$ & 3 & 2 \\
\hline SD & 5 & $2(0 / 2)$ & 3 & 0 \\
\hline PD & 6 & $4(0 / 4)$ & 2 & 0 \\
\hline NE & 8 & $4(1 / 3)$ & 1 & 3 \\
\hline ORR $(\%)^{\mathrm{e}}$ & 36.7 & $33.3(80.0 / 10.0)$ & 40.0 & 40.0 \\
\hline \multicolumn{5}{|l|}{ Main anti-MTB chemotherapy, $\mathrm{n}$} \\
\hline 2HREZ/7HR ${ }^{f}$ & 15 & $8(3 / 5)$ & 4 & 3 \\
\hline $6 \mathrm{HRE} / 6 \mathrm{HR}^{9}$ & 7 & $2(0 / 2)$ & 4 & 1 \\
\hline Levofloxacin-based & 8 & $5(2 / 3)$ & 2 & 1 \\
\hline Duration of anti-MTB treatment (days), median (range) & $275.0(72-637)$ & $\begin{array}{l}274.0(90-469)[274(183-469) / 255 \\
(90-310)]\end{array}$ & $259.0(72-539)$ & $368.0(273-637)$ \\
\hline Duration of concurrent chemotherapy (days), median (range) & $157.5(13-408)$ & $\begin{array}{l}117.0(20-245)[93(20-207) / 121 \\
(48-245)]\end{array}$ & $168.5(13-408)$ & $155.0(51-376)$ \\
\hline MTB treatment outcomes, $\mathrm{n}(\%)$ & All & LC & CRC & Other \\
\hline Cured & $20(66.7)$ & $10(66.7)$ & $6(60.0)$ & $4(80.0)$ \\
\hline Completed & $1(3.3)$ & 0 & $1(10.0)$ & 0 \\
\hline Died & $9(30)$ & $5(33.3)$ & $3(30.0)$ & $1(20.0)$ \\
\hline Failed & 0 & 0 & 0 & 0 \\
\hline Not evaluated & 0 & 0 & 0 & 0 \\
\hline Success $(\%)^{\mathrm{h}}$ & 70.0 & 66.7 & 70.0 & 80.0 \\
\hline
\end{tabular}

Abbreviations $C l$ confidence interval, $C R$ complete response, $C R C$ colorectal cancer, $E$ ethambutol, EGFR-TKI epidermal growth factor receptor-tyrosine kinase inhibitor, $H$ isoniazid, $L C$ lung cancer, MTB Mycobacterium tuberculosis, NE not evaluable, $O R R$ overall response rate, $P D$ progressive disease, $P R$ partial response, $R$ rifampicin, $S D$ stable disease, $Z$ pyrazinamide

${ }^{a}$ Two cytotoxic agents combined with targeted therapy (bevacizumab or trastuzumab)

${ }^{\mathrm{b}}$ Two cytoxic agents

'Erlotinib

${ }^{\mathrm{d}}$ Single cytoxic agent (S-1, vinorelbine, or pemetrexed)

${ }^{e} \mathrm{CR}+\mathrm{PR}$

fDaily drug combination containing HREZ for 2 months, followed by daily HR for 7 months

${ }^{9}$ Daily drug combination containing HRE for 6 months, followed by HR for 6 months

${ }^{\mathrm{h}}$ Cured + completed

0.7, 20.8, and 26.5\%, respectively. [19] The success rate of anti-MTB chemotherapy in this study may have been higher than previous studies $[18,19]$, possibly because of no loss to follow-up and no unevaluated patient in the present study. However, it is important that there were no $M T B$-related treatment failures in our study, suggesting that cancer patients with active MTB would be able to tolerate anti-MTB chemotherapy while receiving anti-CCT as well as non-cancer patients with active $M T B$.
Conversely, the ORRs for all patients combined, LC patients, CRC patients, and other malignancy patients were $36.7,33.3,40.0$, and $40.0 \%$, respectively.

In 15 LC patients, all 5 patients with SCLC received intensive cytotoxic treatment (carboplatin plus etoposide) as first line chemotherapy (Table 3) with a high response rate $(80 \%)$, which is similar to the response rate $(73 \%)$ of similar treatment regimen in the elderly patient or those at poor risk with extensive disease [20]. On the other hand, $10 \%$ of response rate in 10 NSCLC patients 
Table 4 Concurrent Chemotherapy-Related Adverse Events

\begin{tabular}{|c|c|c|c|c|c|c|c|c|}
\hline \multirow[t]{3}{*}{$\overline{\mathrm{NCl}-\mathrm{CTC}^{\mathrm{a}} \text { (Grade) }}$} & \multicolumn{8}{|c|}{ Patients } \\
\hline & \multicolumn{2}{|l|}{ All } & \multicolumn{2}{|l|}{ LC } & \multicolumn{2}{|l|}{ CRC } & \multicolumn{2}{|c|}{ Other } \\
\hline & \multicolumn{2}{|c|}{$(n=28)$} & \multicolumn{2}{|c|}{$\overline{\left(n=13^{b}\right)}$} & \multicolumn{2}{|c|}{$(n=10)$} & \multicolumn{2}{|c|}{$(n=5)$} \\
\hline Adverse event & $1-2$ & $\geq 3$ & $1-2$ & $\geq 3$ & $1-2$ & $\geq 3$ & $1-2$ & $\geq 3$ \\
\hline \multicolumn{9}{|c|}{ Hematological toxicity, number of cases } \\
\hline Leukocytopenia & 7 & 8 & 4 & 6 & 3 & 1 & 2 & 1 \\
\hline Neutropenia & 5 & 14 & 1 & 9 & 2 & 3 & 2 & 2 \\
\hline Anemia & 20 & 2 & 9 & 2 & 8 & 0 & 3 & 0 \\
\hline Thrombocytopenia & 3 & 3 & 1 & 2 & 1 & 1 & 1 & 0 \\
\hline Lymphopenia & 13 & 10 & 4 & $8^{*}$ & 7 & 1 & 2 & 1 \\
\hline
\end{tabular}

Non-hematological toxicity, number of cases

$\begin{array}{lllllllll}\text { AST/ALT elevation } & 12 & 0 & 4 & 0 & 6 & 0 & 2 & 0 \\ \text { Interstitial pneumonitis } & 0 & 1^{\mathrm{c}} & 0 & 1^{\mathrm{c}} & 0 & 0 & 0 & 0 \\ \text { Colitis } & 0 & 1^{\mathrm{d}} & 0 & 0 & 0 & 0 & 0 & 1^{\mathrm{d}} \\ \text { Anaphylaxis } & 0 & 2^{\mathrm{e}} & 0 & 0 & 0 & 2^{\mathrm{e}} & 0 & 0 \\ \text { Hemorrhage } & 1^{\mathrm{f}} & 1^{\mathrm{g}} & 1^{\mathrm{f}} & 0 & 0 & 1^{\mathrm{g}} & 0 & 0\end{array}$

Abbreviations CRC colorectal cancer, $L C$ lung cancer

${ }^{a} \mathrm{NCl}-\mathrm{CTC}$ : National Cancer Institute Common Toxicity Criteria

${ }^{b}$ In two of 15 patients, laboratory data was lost

'Death caused by pemetrexed-induced pneumonitis

dPseudomembranous colitis due to Clostridium difficile infection

e Oxaliplatin- and cetuximab-induced anaphylaxis

${ }^{f}$ Nasal bleeding

${ }^{\mathrm{g}}$ Hemoptysis

*: $P<0.005$

seems to be low. This may be because 6 (60.0\%) of 10 NSCLC patients had received second-line or later anti-CCT after starting anti-MTB chemotherapy.

ORR in CRC patients with $M T B$ was $40 \%$ which was similar to other 1st line combined chemotherapy regimens without molecular targeted therapy in previous reports [21, 22].

Although there were no significant differences in patient characteristics between the LC, CRC, and other malignancy groups, the diagnosis of $M T B$ was more frequently reached during chemotherapy in LC patients when compared to patients with other types of malignancies. If LC and pulmonary $M T B$ coexist in the lungs, a diagnosis of $M T B$ becomes more difficult. Therefore, $M T B$ diagnosis tends to be delayed in these patients.

In a Japanese retrospective study, [23] 247 (28.7\%) of 861 patients who received MTB treatment experienced adverse events. The frequency of adverse events with $M T B$ treatment alone, prior to the initiation of concurrent chemotherapy, in our study was equal to that of the a previous study. [23] Besides, upon starting concurrent chemotherapy in LC and CRC, we did not expect any differences in the prevalence of adverse events between this study and previous studies [20-22] except for liver dysfunction which may be correlated with MTB treatment.

Concurrent chemotherapy-related toxicities were generally acceptable except death caused by pemetrexed-induced pneumonitis. Grade 3 lymphopenia and higher were significantly more frequent in LC compared to other malignancies. The treatment related lymphopenia may affect poor prognosis in LC patients through decreased immune system shown in previous study [24].

Increased multi-drug resistance and extensive drug resistance among strains of $M T B$ is becoming a serious problem worldwide. Hattori et al. reported that 171
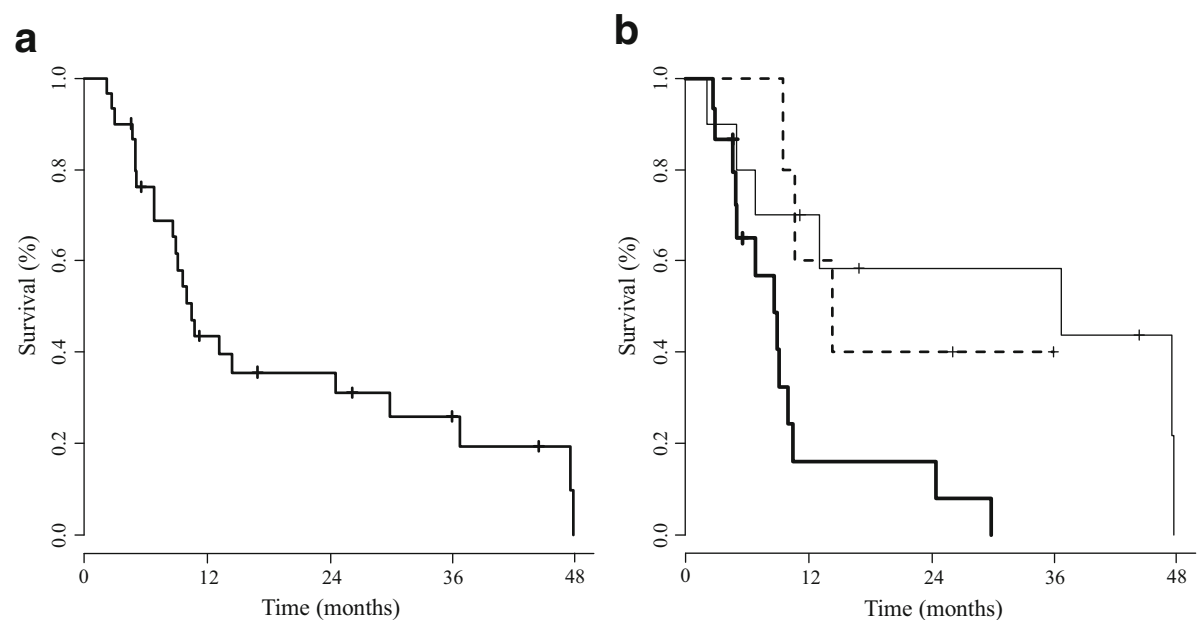

Fig. 2 (a) Kaplan-Meier curve of overall survival for 30 cancer patients with active Mycobacterium tuberculosis who received concurrent chemotherapy between January 1, 2006 and February 29, 2016. The median OS in all patients was 10.5 (8.7-36.7; 95.0\% confidence interval) months (b) Kaplan-Meier curves of overall survival according to cancer type. Lung Cancer patients are represented by the thick solid line, Colorectal Cancer patients are represented by the thin solid line, and patients with Other malignancies, are represented by the dashed line. The median OS according to the type of malignancy were 8.7 (4.7-10.0), 36.7 (minimum 2.2), and 14.4 (minimum 9.6) months for Lung Cancer, Colorectal Cancer, and Other malignancies, respectively 
patients $(0.2 \%)$ in Japan were diagnosed with multi-drug resistant $M T B$, and 48 of these (28.1\%) were foreigners [25]. Although the number of multi-drug resistant MTB cases in Japan is low, Osaka city and Osaka prefecture have the highest prevalence of tuberculosis in Japan (34.4 and 18.2 per 100,000 individuals in 2015 , respectively) [26]. Hence, we are exceedingly cautious of an increase in multi-drug resistant $M T B$. In our institution, anti-CCT was suspended as far as possible until the results of the sensitivity test were known.

Given the benefits of a short duration from MTB diagnosis to the turnaround of results from sensitivity test, MGIT [5] is recommended for culturing mycobacterial organisms.

In this study, $2(8.0 \%)$ of 25 patients had resistant $M T B$ to $\mathrm{H}$ and streptomycin and $\mathrm{H}$ and $\mathrm{Z}$, respectively, but there was no patient with multi-drug resistant MTB. In National-wide survey in Japan [27], the frequencies of drug-resistant isolates from new cases were as follows $8.5 \%$ to any drug which was similar to this study.

Poor PS, extensively drug-resistant MTB (XDR), and severe organ dysfunction were the basic contra-indications for concurrent chemotherapy. However, targeted therapy including EGFR-TKI may be used in selected patients despite a poor PS. Furthermore, concurrent chemotherapy may be initiated in patients with rapidly progressive cancer without waiting the results of $M T B$ sensitivity test, even if the MTB is later categorized as $X D R$. Therefore, there may be no strict contra-indications for concurrent chemotherapy. However, since $6(20 \%)$ of 30 patients experienced adverse events while receiving MTB treatment alone until concurrent chemotherapy, awaiting the result of sensitivity test will be important not only for excluding $X D R$ but also to evaluate the adverse events of $M T B$ treatment alone.

Concurrent use of $\mathrm{R}$, which induces CYP3A4 and CYP2C8 $[28,29]$, may weaken the clinical efficacy of some anti-CCT agents. In clinical practice, we attempt to not administer concurrent chemotherapy along with Erlotinib, Irinotecan, or Pclitaxel and HRE regimen as much as possible. Therefore, a better choice would be X-based regimen or other regimens without rifampicin.

The limitations of this study include its retrospective design and relatively small sample size. It remains unclear whether the findings of this study could be generalized for hematological malignancies or solid malignancies except LC and CRC, or expanded to other institutions. Therefore, a prospective multi-institutional study to evaluate the efficacy and safety of concurrent anti-CCT and anti-MTB chemotherapy in patients with various types of solid tumors and an active $M T B$ infection is warranted.

\section{Conclusions}

Concurrent anti-CCT and anti-MTB chemotherapy is effective and safe for treating cancer patients with active MTB.

\section{Abbreviations}

Anti-CCT: Anti-cancer chemotherapy; ATS: American Thoracic Society; CC: Concurrent chemotherapy; CCA: Cholangiocellular carcinoma;

CCT: Cancer chemotherapy; Cl: Confidence interval; CR: Complete response; CRC: Colorectal cancer; CT: Computed tomography; E: Ethambutol;

GC: Gastric cancer; H: Isoniazid; IDSA: Infectious Diseases Society of America; LAMP: Loop-mediated isothermal amplification; LC: Lung cancer; MDR-

TB: Multi-drug resistant Mycobacterium Tuberculosis; MGIT: Mycobacteria Growth Indicator Tube; MST: Median survival time; MTB: Mycobacterium tuberculosis; NE: Not evaluable; NSCLC: Non-small cell lung carcinoma; PCR: Polymerase chain reaction; PD: Progressive disease; PR: Partial response; XDR: Extensively drug-resistant MTB; Z: Pyrazinamide

\section{Acknowledgements}

The authors thank Editage (www.editage.jp) for English language editing and a part donation to the Osaka Prefectural Hospital Organization (Osaka, Japan) for writing our manuscript.

\section{Funding}

This study had no funding source.

\section{Availability of data and materials}

We cannot share the detailed data, because the Institutional Review Board of the Osaka Habikino Medical Center has not approved it.

\section{Authors' contributions}

$\mathrm{TH}, \mathrm{HS}, \mathrm{NO}, \mathrm{YT}$, and TN planned and designed the study. TH, YT, YH, SH and TN collected data of Bacteriological Examinations, and adverse effects and outcomes of MTB treatment; TH, AT, TS, NM, HS, NO, SA, MF, YK, KS, AN, and SM collected data of adverse effects and outcomes of cancer treatment. TH and HS performed the statistical analyses. All authors have been involved in drafting the manuscript or revising it critically for important intellectual content and approved the final manuscript.

\section{Ethics approval and consent to participate}

Ethics and publication of this study was approved by the Institutional Review Board of the Osaka Habikino Medical Center (formerly the Osaka Prefectural Medical Center for Respiratory and Allergic Diseases) (Osaka, Japan) on January 30, 2017 (approval no.: 808-1). The board waived the requirement for informed written consent due to the anonymous nature of the data and the fact that this research presented no risk of exposure to the subjects. Research was conducted in accordance with the 1964 Declaration of Helsinki and its later amendments.

\section{Consent for publication}

Not applicable.

\section{Competing interests}

T.H. has received honoraria and research funding from Ono Pharmaceutical Co. Ltd. (Osaka, Japan), Lilly Japan Co. Ltd. (Hyogo, Japan), AstraZeneca Co. Ltd. (Osaka, Japan), Taiho Pharmaceutical Co. Ltd. (Tokyo, Japan), Chugai Pharmaceutical Co. Ltd. (Tokyo, Japan), and MSD Oncology Co. Ltd. (Tokyo, Japan). T.H. received honoraria from Kyowa-Hakko Kirin and Boehringer Ingelheim. T.H. received research funding from Merck Serono Co. Ltd. (Tokyo, Japan). The remaining authors (Y.T., Y.H., S.H., A.T., T.S., N.M., H.S., N.O., S.A., M.F., Y.K., K.S., A.N., S.M., and T.N.) declare no competing interests.

\section{Publisher's Note}

Springer Nature remains neutral with regard to jurisdictional claims in published maps and institutional affiliations.

\section{Author details}

'Department of Thoracic Oncology, Osaka Habikino Medical Center, 3-7-1 Habikino, Habikino City, Osaka 583-8588, Japan. ${ }^{2}$ Departments of Clinical Laboratory, Osaka Habikino Medical Center, 3-7-1 Habikino, Habikino City, 
Osaka 583-8588, Japan. ${ }^{3}$ Departments of Infectious Diseases, Osaka Habikino Medical Center, 3-7-1 Habikino, Habikino City, Osaka 583-8588, Japan. ${ }^{4}$ Departments of Gynecology, Osaka Habikino Medical Center, 3-7-1 Habikino, Habikino City, Osaka 583-8588, Japan. ${ }^{5}$ Departments of Breast Surgery, Osaka Habikino Medical Center, 3-7-1 Habikino, Habikino City, Osaka 583-8588, Japan. ${ }^{6}$ Departments of Thoracic Surgery, Osaka Habikino Medical Center, 3-7-1 Habikino, Habikino City, Osaka 583-8588, Japan. ${ }^{7}$ Departments of Gastroenterological Surgery, Osaka Habikino Medical Center, 3-7-1 Habikino, Habikino City, Osaka 583-8588, Japan.

Received: 19 August 2017 Accepted: 2 October 2018 Published online: 12 October 2018

\section{References}

1. WHO: Global tuberculosis report 2017. [http://www.who.int/tb/country/ data/download/en/]. Accessed 1 Aug 2018.

2. Hirashima T, Nagai T, Shigeoka H, Tamura Y, Yoshida H, Kawahara K, et al. Comparison of the clinical courses and chemotherapy outcomes in metastatic colorectal cancer patients with and without active Mycobacterium tuberculosis or Mycobacterium kansasii infection: a retrospective study. BMC Cancer. 2014;14:770.

3. Steingart KR, Henry M, Ng V, Hopewell PC, Ramsay A, Cunningham J, et al. Fluorescence versus conventional sputum smear microscopy for tuberculosis: a systematic review. Lancet Infect Dis. 2006;6:570-81.

4. Notomi T, Okayama H, Masubuchi H, Yonekawa T, Watanabe K, Amino N, et al. Loop-mediated isothermal amplification of DNA. Nucleic Acids Res. 2000; 28:E63.

5. Bemer P, Palicova F, Rusch-Gerdes S, Drugeon HB, Pfyffer GE. Multicenter evaluation of fully automated BACTEC mycobacteria growth Indicator tube 960 system for susceptibility testing of Mycobacterium tuberculosis. J Clin Microbiol. 2002;40:150-4

6. Kudoh S, Kudoh T. A simple technique for culturing tubercle bacilli. Bull World Health Organ. 1974;51:71-82.

7. Yajko DM, Madej JJ, Lancaster MV, Sanders CA, Cawthon VL, Gee B, et al. Colorimetric method for determining MICs of antimicrobial agents for Mycobacterium tuberculosis. J Clin Microbiol. 1995;33:2324-7.

8. Blumberg HM, Burman WJ, Chaisson RE, Daley CL, Etkind SC, Friedman LN, et al. American thoracic society/Centers for Disease Control and Prevention/ Infectious Diseases Society of America: treatment of tuberculosis. Am J Respir Crit Care Med. 2003;167:603-62.

9. WHO: Definitions and reporting framework for tuberculosis - 2013 revision (updated December 2014) A.2.1 Treatment outcomes for TB patients [http://apps.who.int/iris/bitstream/handle/10665/79199/9789241505345_eng. pdf;jsessionid]. Accessed 1 Aug 2018.

10. JLCS Guidelines 2015 for the Treatment of Lung Cancer [https://www. haigan.gr.jp/modules/guideline/]. Accessed 1 Aug 2018.

11. Watanabe T, Itabashi M, Shimada Y, Tanaka S, Ito Y, Ajioka Y, et al. Japanese Society for Cancer of the Colon and Rectum (JSCCR) guidelines 2014 for treatment of colorectal cancer. Int J Clin Oncol. 2015;20:207-39.

12. Japanese gastric cancer treatment guidelines [http://www.jgca.jp/guideline. html]. Accessed 1 Aug 2018.

13. Tozaki M, Kuroki Y, Kikuchi M, Kojima Y, Kubota K, Nakahara H, et al. The Japanese breast Cancer society clinical practice guidelines for screening and imaging diagnosis of breast cancer, 2015 edition. Breast Cancer. 2016;23: 357-66.

14. Furuse J, Takada T, Miyazaki M, Miyakawa S, Tsukada K, Nagino M, et al. Guidelines for chemotherapy of biliary tract and ampullary carcinomas. J Hepato-Biliary-Pancreat Surg. 2008;15:55-62.

15. Therasse P, Arbuck SG, Eisenhauer EA, Wanders J, Kaplan RS, Rubinstein L, et al. New guidelines to evaluate the response to treatment in solid tumors. J Natl Cancer Inst. 2000;92:205-16.

16. Trotti A, Colevas AD, Setser A, Rusch V, Jaques D, Budach V, et al. CTCAE v3. 0 : development of a comprehensive grading system for the adverse effects of cancer treatment. Semin Radiat Oncol. 2003;13:176-81.

17. Kaplan E, Meier P. Nonparametric estimation from incomplete observations. J Am Stat Assoc. 1958;53:457-81.

18. WHO; Download treatment outcomes [http://www.who.int/tb/country/data/ download/en/]. Accessed 1 Aug 2018.

19. Katsuda N, Hirosawa T, Reyer JA, Hamajima N. Roles of public health centers (Hokenjo) in tuberculosis control in Japan. Nagoya J Med Sci. 2015;77:19-28.
20. Okamoto H, Watanabe K, Kunikane H, Yokoyama A, Kudoh S, Asakawa T, et al. Randomised phase III trial of carboplatin plus etoposide vs split doses of cisplatin plus etoposide in elderly or poor-risk patients with extensive disease small-cell lung cancer: JCOG 9702. Br J Cancer. 2007;97:162-9.

21. Van Cutsem E, Kohne CH, Hitre E, Zaluski J, Chang Chien CR, Makhson A, et al. Cetuximab and chemotherapy as initial treatment for metastatic colorectal cancer. N Engl J Med. 2009;360:1408-17.

22. Douillard JY, Siena S, Cassidy J, Tabernero J, Burkes R, Barugel M, et al. Randomized, phase III trial of panitumumab with infusional fluorouracil, leucovorin, and oxaliplatin (FOLFOX4) versus FOLFOX4 alone as first-line treatment in patients with previously untreated metastatic colorectal cancer: the PRIME study. J Clin Oncol. 2010;28:4697-705.

23. Yamamoto $Y$, Hasegawa $Y$, Ogawa K. Retrospective cohort study of risk factors for adverse effects of antituberculous therapy. Kekkaku. 2011;86:499-507.

24. Grossman SA, Ellsworth S, Campian J, Wild AT, Herman JM, Laheru D, et al. Survival in patients with severe lymphopenia following treatment with radiation and chemotherapy for newly diagnosed solid tumors. J Natl Compr Cancer Netw. 2015;13:1225-31.

25. Hattori T, Kobayashi N, Nagai H, Chagan-Yasutan H, Telan E, Solante MB. Nationwide HIV-, MDR-TB survey in Japan and collaborative study in the Philippines. Int J Mycobacteriol. 2016;5(Suppl 1):S18-9.

26. Statistic of TB 2015 [http://www.jata.or.jp/rit/ekigaku/en/statistics-of-tb/]. Accessed 1 Aug 2018

27. Tuberculosis Research Committee TJ. Nationwide survey of anti-tuberculosis drug resistance in Japan. Int J Tuberc Lung Dis. 2015;19:157-62.

28. Rakhit A, Pantze MP, Fettner S, Jones HM, Charoin JE, Riek M, et al. The effects of CYP3A4 inhibition on erlotinib pharmacokinetics: computer-based simulation (SimCYP) predicts in vivo metabolic inhibition. Eur J Clin Pharmacol. 2008;64:31-41.

29. Kajosaari LI, Laitila J, Neuvonen PJ, Backman JT. Metabolism of repaglinide by CYP2C8 and CYP3A4 in vitro: effect of fibrates and rifampicin. Basic Clin Pharmacol Toxicol. 2005;97:249-56.

Ready to submit your research? Choose BMC and benefit from:

- fast, convenient online submission

- thorough peer review by experienced researchers in your field

- rapid publication on acceptance

- support for research data, including large and complex data types

- gold Open Access which fosters wider collaboration and increased citations

- maximum visibility for your research: over $100 \mathrm{M}$ website views per year

At BMC, research is always in progress.

Learn more biomedcentral.com/submissions 